\title{
Interrelación entre disociación, absorción y propensidad a la fantasía con experiencias alucinatorias en poblaciónes no psicóticas
}

Alejandro Parra

La hipótesis del continuo de la psicosis sugiere que síntomas tales como las alucinaciones pueden ser parte de la conciencia normal de la población general. En este estudio examinamos tres factores que podrían estar relacionados con la experiencia alucinatoria: la propensidad a la fantasía, la absorción psicológica y las experiencias disociativas. Examinamos una muestra de 650 estudiantes universitarios de ambos sexos (24\% varones y 76\% mujeres; edad $=17-57$ años) $y$ encontramos diferencias significativas en absorción $(r=.61 p<.001) y$ -aunque algo menor- propensidad a la fantasía $(r=.54 ; p<.001)$, y disociación $(r=.55 ; p<.001)$ con el puntaje total de alucinación. Dada la consistencia de nuestro descubrimiento respecto a la absorción, la disociación y la propensidad a la fantasía tenemos una validación de las experiencias alucinatorias en población normal.

\section{Experiencia alucinatoria / absorción psicológica / propensidad a la fantasía / experiencias disociativas.}

\section{Correlations between dissociation, absorption and fantasy proneness with hallucinatory experiences in healthy population}

The continuum hypothesis of psychosis suggests that symptoms as well as hallucinations can make part of normal consciousness in the general population. In the present study we examined three factors hypothetically related to hallucinatory experiences: fantasy proneness, psychological absorption and dissociative experiences. We surveyed a sample of 650 university students of both sexes (24\% males and $76 \%$ females; ages $17-57$ years) and found significant differences in absorption $(r=.61 p<.001)$ and-although somewhat less-fantasy proneness $(r=.54 ; p<.001)$ and dissociation $(r=.55 ; p<.001)$ with the total hallucination score. Given the consistency of our findings with regard to absorption, dissociation, and fantasy proneness, we conclude a validation of hallucinatory experiences in the normal population.

Hallucinatory experiences / psychological absorption / fantasy proneness / dissociative experiences 


\section{INTRODUCCIÓN}

Hay un sustancial número de artículos en la literatura psiquiátrica y psicológica acerca de individuos en la población general que dicen haber tenido experiencias alucinatorias.

Sidgwick (1894), quien investigó la incidencia de los reportes alucinatorios en 17 mil individuos de diez países, y West (1948), con una muestra más pequeña, encontraron que aproximadamente el $10 \%$ de estas muestras estaba relacionado con el uso de drogas o con presencia de psicopatología.

Al igual que Galton (1883) y James (1892) en el siglo XIX, Sarbin y Juhasz (1967) sugieren que las alucinaciones deben ser entendidas como resultado de un proceso mental normal, conocido como modelo psicológico de alucinación.

Una versión particular de este modelo es defendido por Slade y Bentall (1988), que desarrollaron la "hipótesis de continuidad" de la experiencia alucinatoria. De acuerdo con estos autores, las alucinaciones están relacionadas con experiencias de la conciencia normal, como las ensoñaciones diurnas vívidas e intensas (Launay \& Slade, 1981).

Utilizando información de 300 individuos, Launay y Slade construyeron un instrumento que contenía 12 declaraciones sobre pensamientos y fantasías vívidas y alucinaciones visuales y verbales (véase Bentall, 1990, 2000).
Posey y Losch (1983) estudiaron una muestra de 375 estudiantes utilizando un cuestionario que contenía descripciones de experiencias verbales en la población general y descubrieron que el $71 \%$ indicaba haber experimentado algo similar al menos una vez por semana. Barret y Etheridge (1992) examinaron una muestra de 576 estudiantes utilizando una versión modificada de la escala de alucinación de Barret, que permitió una estimación más precisa de cuán a menudo estas experiencias ocurrían en cada individuo en particular. Más del 10\% había tenido la experiencia al menos una vez al mes, y el porcentaje general de individuos que tuvieron una experiencia era virtualmente el mismo que el de Posey y Losch (1983).

Barret y Etheridge observaron que el $45 \%$ había ocurrido con la frecuencia de una vez al día en un mes. No hubo una correlación significativa entre las alucinaciones verbales y la deseabilidad social, así como tampoco diferencia alguna entre las alucinaciones verbales entre alucinadores y no alucinadores en las escalas de psicopatología. Las alucinaciones verbales parecen ser un componente normal, y relativamente frecuente para gran parte de la población general.

Que una porción substancial de estudiantes indique haber tenido experiencias que podrían llamarse alucinaciones verbales representa un descubrimiento potencialmente importante. Po- 
dría ser, como sugiere la hipótesis del continuo, que estas experiencias sean parte de la conciencia normal para la población general.

Las alucinaciones generalmente frecuentes son un indicador de psicopatología (Hoffman, 1986). No cabe duda de que estos estudios producen un sinnúmero de cuestiones empíricas y teóricas importantes que deben ser resueltas. Wilson y Barber (1981, 1983), por ejemplo, descubrieron que individuos con alta susceptibilidad hipnótica tendían a tener fantasías vívidas y sueños diurnos, al punto de que no podían imaginar su vida sin ellos. Las fantasías tenían propiedades alucinatorias.

Investigaciones recientes que utilizaron el constructo de "propensión a la fantasía" (Lynn \& Rhue, 1988) confirman la sospecha de que la propensión a la fantasía y la absorción están altamente correlacionadas $(r=.70$ en el artículo de Lynn y Rhue). Tellegen (1982) ha utilizado una escala de absorción para investigar dos aspectos de experiencias relacionadas conceptualmente con las experiencias alucinatorias, como la sinestesia y el eidetisismo (Glicksohn, 1992, 1999; Rader y Tellegen, 1987) que involucran tanto a la imaginación (Crawford, 1993-1994; Glicksy et al., 1991) como a las experiencias subjetivas anómalas y las creencias (Glickson, 1990).

Según Tellegen (1982), la absorción es un rasgo de la personalidad que permite que un individuo se implique en una variedad de experiencias mediante la imaginación. Las personas que tienen absorción tienen imaginación intensa y vívida, y tienen experiencias sensoriales e imaginativas al punto de perder su sentido del yo (un ejemplo común es ver una película e involucrarse tanto en esta que se pierde el contexto que lo rodea, incluso si otro le habla). Si la absorción es un factor de predisposición para las experiencias alucinatorias, esto tiene algunas implicaciones para la psicología cognitiva. Sujetos con alta disposición para la absorción a menudo han tenido también alguna experiencia traumática en la infancia (Lynn \& Rhue, 1988) debido a una sensibilidad premórbida que intensifica las experiencias subjetivas, como las alucinaciones (Merckelbach et al., 2000; Freyd, Martorello, Alvarado, Hayes \& Christman, 1998; Glickson, 2001; Glickson \& Barret, 2003). Como sugieren Berenbaum, Kerns \& Raghavan (2000: 39): “Aquellos individuos con niveles elevados de absorción... están en riesgo de tener experiencias anómalas debido a que quizás traten de tenerlas intencionalmente, o quizás estén más dispuestos a explorar aspectos de sus mundos fenomenológicos que otras personas no pueden explorar".

Según el DSM-IV, la disociación se entiende como una "ruptura de las funciones de la conciencia que normalmente están integradas a la memoria, la identidad y la percepción del entorno" (American Psychiatric Association, 
1994: 477). Hilgard (1986) define la disociación como una "manera especial de la conciencia de mantener conectados eventos que de lo contrario estarían divididos entre sí" (p. 80). De este modo, los procesos disociativos incorporan la idea de que la mente está compuesta por módulos que constantemente están interactuando en varios niveles para generar la unidad de la conciencia. En circunstancias normales, la integración de estos módulos ocurre a la perfección, operando como una entidad homogénea. Pero, en el caso de la disociación, los sistemas cognitivos comienzan a desconectarse entre sí hasta que se desintegran funcionalmente. Por último, para Cardeña (1994), es "el dominio de la disociación" (p. 15) el que identifica el núcleo de la constelación de la disociación y sus fenómenos relacionados. Otras definiciones adoptan una perspectiva psicodinámica y consideran la disociación un mecanismo de defensa inconsciente.

Los procesos disociativos pueden ocurrir en contextos normales, como también en contextos patológicos. En la categoría de la disociación "normal" encontramos las experiencias como "experiencias fuera del cuerpo", el automatismo, y la absorción hipnótica y cognitiva (Cardeña, 1994; Kihlstrom \& Hoyt, 1990; Putnam, 1997; Steinberg, 1995). De hecho, actualmente algunos antropólogos y psicólogos transculturales creen que una amplia variedad de experiencias religiosas y de posesión son fenómenos disociativos normales (Bourguignon, 1976; Castillo, 1997; Golub, 1995; Goodman, 1988). La cuestión aquí es determinar cuándo un estado disociativo se desvía de lo normal o se convierte en patológico, o sea, cuándo se presentan tendencias disociativas que causan malestar o sufrimiento innecesario al individuo. Esta desviación se comprende mejor solo si estas experiencias ocurren contextualmente, esto es, en relación con influencias culturales, religiosas o sociales (Martínez Taboas, 1999, 2001). Entonces, cuando las experiencias disociativas son normales o patológicas, es importante comprender su construcción social, y la pluralidad de los significados culturales que se les puede atribuir (Martínez Taboas, 1999, 2001). Existen varios tipos de fenómenos disociativos, como la amnesia disociativa, las fugas disociativas, la despersonalización/desrealización y el trastorno de identidad disociativa (conocida también como trastorno de personalidad múltiple) (Khilstrom, 1994).

En efecto, algunos especialistas coinciden en que las experiencias disociativas operan en un continuo en cuyo extremo están los estados disociativos normales (autohipnosis), hasta estados patológicos (amnesia disociativa y despersonalización) (Ross, 1989). Otros investigadores proponen que la disociación no debería ser conceptualizada como dimensional, sino estudiada de manera categorial. Por ejemplo, hay 
estudios taxométricos que sugieren que existen categorías cualitativamente diferentes de procesos disociativos, algunas más patológicas que otras (Waller, Putnam \& Carlson, 1996). El modelo "taxonómico" de disociación supone que la disociación normal y la patológica son de diferente tipo, y que este último se relaciona con experiencias que son profundamente diferentes entre sí, o que nunca o rara vez aparecen, y de aquellas experiencias disociativas normales que tienen las personas. De acuerdo con este modelo, los ejemplos típicos de disociación patológica serían la fuga disociativa o el trastorno de identidad disociativo. En la actualidad, sin embargo, ni la validez del modelo "taxonómico" ni la del modelo "dimensional de continuidad" han sido concluyentes (Putnam, 1997).

Algunos investigadores han tratado de determinar cuál es la naturaleza de los fenómenos disociativos en individuos normales. Muchos estudios han descubierto que los individuos seleccionados de una población "normal" por lo general tienen puntajes más altos en el factor absorción/imaginación del Dissociative Experiences Scale (DES), y algo menos en despersonalización/desrealización y amnesia (véanse Carlson, 1994; Ray, 1996). En otras palabras, muchas personas normales pueden experimentar situaciones en las cuales su conciencia parece dividirse; donde existe un procesamiento de percepciones, pensamientos, sentimientos y acciones sin representación consciente. Tales circunstancias pueden implicar cogniciones y conductas percibidas como involuntarias o que ocurren sin ser accesibles a la conciencia. Este tipo de disociación no es patológica. Estos procesos mentales no conscientes no se limitan solo a los que surgen de conductas sexuales o agresivas primitivas; de hecho, no tiene por qué estar motivada por mecanismos de defensa contra conflictos intrapsíquicos o experiencias traumáticas.

\section{HIPÓTESIS}

El objetivo de este estudio es determinar en qué grado las experiencias alucinatorias se relacionan con las modalidades sensoriales de experiencias alucinatorias. Se examinarán tres hipótesis, según las cuales las seis modalidades sensoriales de experiencia alucinatoria correlacionarán en términos de intensidad de la disociación, la absorción y la propensidad a la fantasía.

\section{MÉTOdo}

\section{Participantes}

La muestra se compone de 650 estudiantes universitarios de psicología y está constituida por individuos de ambos sexos, 156 varones (24\%) y 494 mujeres $(76 \%)$, cuyo rango etario es de 17 a 57 años (Media $=25,57$; $\mathrm{SD}=7,23 ;$ Media varones $=26,07$; $\mathrm{SD}=6,59 ;$ Media mujeres $=25,39$, 
$\mathrm{SD}=7,42)$ de la Universidad Abierta Interamericana.

\section{Instrumentos}

Basados en los cuestionarios creados por Barrett (Barrett y Etheridge, 1992; Barrett, 1993; Barrett y Etheridge, 1994) y Launay y Slade (Escala de Alucinaciones Launay-Slade -Revisada (LSHS-R) (Launay \& Slade, 1981, se diseñó una escala de 38 ítems para medir la predisposición a las alucinaciones, particularmente sobre individuos no psicóticos, en siete modalidades sensoriales: auditiva (ítems 1 a 16), visual (ítems 17 a 25), gustativa (ítems 26 a 28), táctiles (ítems 29 a 33), olfativas (ítems 34 a 38) e hipnagógico/hipnopómpicas (H/H) (ítems 12, 25, $28,30,31,33$ y 38$)$. La H/H es una subescala que representa la suma de los ítems correspondientes a cada modalidad sensorial, pero no distingue entre hipnagógica (pasaje de la vigilia al sueño) e hipnopómpica (pasaje del sueño a la vigilia). Cada ítem se responde mediante una escala Likert de cinco puntos, siendo 0 (nunca) y 1 (rara vez), 2 (ocasionalmente), 3 (a menudo) y 4 (muy a menudo). La confiabilidad de la escala con el uso del coeficiente $\alpha$ de Cronbach indicó que produce datos consistentes internamente ( $\alpha$ de Cronbach $=.93$ ); la confiabilidad estimada para las modalidades sensoriales también fue alta (auditivas $=.90$, visuales $=.90$, gustativas $=.72$, táctiles $=.76$, olfativas $=.74 \mathrm{e} \mathrm{H} / \mathrm{H}=.75$, Media $=$
$.80)$. En general, un valor $\alpha$ de Cronbach de .60 es una medida aceptable de confiabilidad (Grady \& Wallston, 1988; Kim \& Mueller, 1978).

- Escala de Absorción de Tellegen (Tellegen \& Atkinson, 1974). Es un test autoadministrable de 34 ítems de respuesta dicotómica (V-F) cuya estabilidad test-retest y consistencia interna ha demostrado ser adecuada (Tellegen, 1982). Ha sido previamente utilizada para investigar varias facetas de experiencias subjetivas, conceptualmente relacionadas, como la imaginación, e incluso brinda una apertura a la experiencia de las alteraciones cognitivas afectivas en una variedad de situaciones (Roche \& McConkey, 1990).

- Escala de Experiencias Disociativas - Revisada (Bernstein \& Putnam, 1986). Es un cuestionario autoadministrable de 28 ítems, con una escala Likert de cero (nada) a 100 (máxima), que permite identificar tanto pacientes con psicopatologías disociativas, además de servir como instrumento de investigación cuantitativa de experiencias disociativas en la población normal. La confiabilidad del cuestionario tiene alta consistencia interna (Frischholz et al, 1992; Ross, Norton \& Anderson, 1988; Pitblado \& Sanders, 1991; Ross, Joshi \& Currie, 1991). En el DES se pide circular un número en un rango de 0 a 100 ("nunca" a "todo el tiempo"). El DES explo- 
ra experiencias disociativas, como absorción, despersonalización y amnesia. El instrumento permite obtener un valor que es el promedio del puntaje total.

- Cuestionario de Propensidad a la Fantasía (Merckelbach, Muris, Horselenberg \& Stougie, 2000). Es un instrumento autoadministrable de 25 ítems de respuesta dicotómica que permite evaluar la tendencia a fantasear. Las respuestas son sumadas y se obtiene un puntaje total. Las puntuaciones altas implican niveles elevados de propensión a la fantasía. Una correlación productomomento de Pearson entre este cuestionario y los puntajes del DES fueron altamente significativas, en un rango de .47 a .63 (Merckelbach, Muris, Horselenberg \& Stougie, 2000). El Creative Experiencies Questionnaire (CEQ) tiene validez predictiva en el sentido de que ciertos individuos (por ejemplo personas con experiencias paranormales, actores, véase Lawrence et al., 1995) tienden a puntuar alto en propensidad a la fantasía más que un grupo control. Recientes estudios también han identificado que eventos de la niñez imaginarios o escribir acerca de eventos ficticios pueden producir seudorrecuerdos en un subgrupo de participantes (por ejemplo Garry et al., 1996; Horselenberg, Merckelbach, Muris, Sijsenaar \& Spaan, 2000). Hay indicado- res de que la propensidad a la fantasía puede contribuir a la susceptibilidad al seudorrecuerdo y generar experiencias alucinatorias (véase Hyman \& Billings, 1998).

\section{Procedimiento}

Aplicamos una técnica de muestreo no probabilística. La muestra comprende estudiantes universitarios de ambos sexos, a partir de los 17 años, e incluye a estudiantes de primero a quinto año de las carreras de psicología de la Facultad de Psicología de la Universidad Abierta Interamericana, residentes en la Ciudad Autónoma de Buenos Aires y Gran Buenos Aires. Se administró en las aulas de las sedes Centro, Almagro, y Campus Sur (Lomas de Zamora) y el segundo año en la Universidad del Salvador (USAL).

El set de tests autoadministrables fue entregado en un sobre A4 a cada estudiante, en forma contrabalanceada, durante una clase teórica. El tiempo promedio para completar los cuestionarios fue de 40 minutos. Los estudiantes recibieron una vaga información del objeto de estudio y se les invitó a participar voluntaria y anónimamente completando los tests, en una única sesión, en días y horarios previamente pactados con los docentes.

El orden de administración de ambas pruebas fue contrabalanceado y el cuestionario de alucinaciones fue presentado bajo el seudotítulo de Cuestionario de Experiencias Psicológicas, 
con lo cual intentamos evitar distorsiones en las respuestas. Los análisis fueron procesados mediante el SPSS 11.5 (en español).

\section{Resultados}

Empleando el análisis de correlación con el estadístico $r$ de Pearson, se encontraron diferencias significativas en absorción con el puntaje total de alucinación $(r=.615 p<.001$, a dos colas $) \mathrm{y}$ -aunque algo menor- propensidad a la fantasía $(r=.546 ; \mathrm{p}<.001)$, lo cual indica que individuos que "alucinan" tienden a puntuar alto en absorción y son más propensos a fantasear. No se encontró una correlación estadísticamente significativa entre disociación con el puntaje total de alucinación, excepto la modalidad auditiva $(r=.554$; $\mathrm{p}<.001$, a dos colas) (véase tabla 1 ).

Finalmente, se realizaron análisis de regresión lineal por el método de pasos sucesivos para valorar hasta qué punto la absorción, la disociación y la propensidad a la fantasía explican la pre- sencia de experiencias alucinatorias. A causa de que las modalidades sensoriales de experiencias alucinatorias mostraban una notable asimetría, lo cual podría distorsionar el análisis, procedimos a normalizar las variables mediante la fórmula de estimación de la proporción de Blom. Para ello se obtuvieron estimaciones de la proporción de casos acumulados por cada rango, y luego se obtuvieron puntuaciones típicas normales que corresponden a tales proporciones. Se incluyeron en la fórmula aquellas variables seleccionadas cuyo coeficiente de correlación sea igual o superior a .30 , respecto de la modalidad sensorial de alucinación. Tomando como variable el criterio tres de las seis modalidades (auditiva, táctil, hipnagógico/hipnopómpica) y el puntaje total de alucinación, nuestros resultados muestran que la absorción (VC auditiva: $\mathrm{R}^{2}=.350$, $\beta=.400, t=10.192, p<.001 ; \mathrm{VC}$ Táctil: $\mathrm{R}^{2}=.194, \beta=.261, t=6.23$ $p<.001)$ y la disociación (VC $\mathrm{H} / \mathrm{H}$ :

Tabla 1

Correlación entre modalidades sensoriales de alucinación y los puntajes de disociación, absorción y propensidad a la fantasía

\begin{tabular}{lcccccccc}
\hline \multicolumn{7}{c}{ Modalidad sensorial } \\
\hline & $\alpha$ & Auditiva & Visual & Gustativa & Táctil & Olfativa & H/H & Total \\
\hline Disociación & .85 & $.554^{*}$ & n.s. & n.s. & n.s. & n.s. & n.s. & n.s. \\
\hline Absorción & .91 & $.597^{*}$ & $.460^{*}$ & $.428^{*}$ & $.449^{*}$ & $.473^{*}$ & $.503^{*}$ & $.615^{\star * *}$ \\
\hline Fantasía & .89 & $.509^{*}$ & $.413^{*}$ & $.401^{*}$ & $.393^{*}$ & $.382^{*}$ & $.455^{*}$ & $.546^{*}$ \\
\hline
\end{tabular}

${ }^{*} p<.001 ; p$ ajustada $=.008$, a dos colas. 
$\mathrm{R}^{2}=.248, \beta=.252, t=6.03, p<.001 ;$ VC: P. Total: $\mathrm{R}^{2}=.393, \beta=.276$, $t=4.90, p<.001$ ) explican el $16 \%$ de la varianza observada en la experiencia alucinatoria. La varianza explicada de la propensidad a la fantasía no ha sido satisfactoria en este análisis.

\section{DISCUSIÓN}

Dadas las características de la muestra y la consistencia de nuestro descubrimiento con respecto a la absorción, la disociación y la propensidad a la fantasía, tenemos una validación de las experiencias alucinatorias en población normal. En efecto, las características de absorción que subyacen a las experiencias alucinatorias radican en que estas pueden ser vistas como formas de disociación. Nuestros hallazgos son apenas el inicio de importantes implicaciones para ser aplicadas en psicología cognitiva.

Recientemente se viene prestando un enorme interés a la investigación del trastorno por estrés postraumático (PTSD, por sus siglas en inglés). Los desastres naturales y otras catástrofes pueden servir como eventos estresores precipitadores (por ejemplo la destrucción de una casa o de una comunidad por una explosión volcánica, huracanes, incendios forestales, terremotos) dan lugar a un tipo de trastorno en la población. El PTSD se define y caracteriza por tres síntomas primarios (Kinzie, 1989): experiencia intrusiva (ima- ginación no inventada), regresión y negación (por ejemplo disociación) y aumento de la excitación (hipervigilancia) (Burges et al., 1988; Horowitz, 1986; Kinzie, 1989) aunque no todos los individuos expuestos a eventos traumáticos desarrollen PTSD, se hace evidente que debe de haber algún elemento que predispone a la personalidad premórbida del sujeto que interactúa con el evento traumático, y esto resulta en un síndrome (Horowitz, 1986). La frecuencia de la ocurrencia de la intrusión y la negación de la experiencia indica claramente su severidad, y coloca a la PTSD firmemente dentro de la categoría de los trastornos disociativos (véanse Bremmer et al., 1992; Horowitz, 1986; Spiegel et al., 1988, Van der Kolk \& Van der Hart, 1989). Los sujetos que padecen de PTSD puntúan alto en el DES en relación con los sujetos de control, o en individuos con fobias y esquizofrénicos (Bernstein \& Putman, 1986).

Janet, que se refiere a la reexperimentación traumática como "crisis sonambulística" (Van der Kolk \& Van der Hart, 1989), está dentro de este contexto. Se puede observar una interrelación entre la hipnosis y la personalidad PTSD (Butler et al., 1997). Se ha estudiado que sujetos PTSD parecen ser altamente hipnotizables (Spiegel, Hunt \& Dondershine, 1988) y que la relación entre la hipnosis y el PTSD se ha documentado en varias culturas y situaciones. Por ejemplo, Sargant (1957, 
1973), descubrió la hiperexcitabilidad de sus pacientes en la Segunda Guerra Mundial (conocida como "neurosis de guerra"), argumentando que esta hiperexcitabilidad resultaba en subsecuentes colapsos psicofisiológicos, acompañado de un estado hipnoide de hipersugestionabilidad. De modo que la susceptibilidad PTSD quizás esté relacionada con la susceptibilidad hipnótica, y esta a su vez relacionada con la absorción (Tellegen \& Atkinson, 1974).

Otro caso es el de los falsos recuerdos o seudorrecuerdos, que han estado bajo estudio desde hace veinte años. Nachson (2001) ha revisado esta literatura-específicamente la que concierne a los recuerdos evocados de abusos sexuales durante la niñez- evaluando la cuestión del autoengaño, lo cual -según su punto de vista- funciona como una "separación entre varios sistemas cognitivos" (p. 2), de modo que el autoengaño y la disociación parecen ser dos caras de una misma moneda (véase Lawrence \& Perry, 1983). Si pensamos que algunas experiencias traumáticas previas lesionan la memoria autobiográfica, entonces deberíamos considerar la absorción como un factor de predisposición o como un factor mediatizador.

En resumen, podemos pensar que aquellos investigadores que estudian la cognición en forma separada de la personalidad cometen un grave error (Messik, 1994: 133). Las investigaciones en el campo de la memoria auto- biográfica, la memoria traumática y los seudorrecuerdos, que ignoren las diferencias individuales en áreas tan vinculadas entre sí como la absorción y la disociación, tendrán necesariamente una severa limitación. Afortunadamente, como lo han demostrado un gran número de artículos (Destun \& Kuiper, 1999; Eisen \& Carlson, 1998; Loftus, 1997; Platt et al., 1998), se pueden tender productivamente puentes entre estos campos de investigación.

\section{REFERENCIAS}

Aleman, A.; Nieuwenstein, M. R.; Bocker, K. B. E. \& De Haan, E. H. F. (2001). Multidimensionality of hallucinatory predisposition: Factor structure of the Launay-Slade Hallucination Scale in a normal sample. Personality and Individual Differences, 30, 287-292.

American Psychiatric Association. (1994). Diagnostic and statistical manual of mental disorders (DSMIV) (4th ed). Washington, DC: Author.

Barrett, T. R. \& Etheridge, J. B. (1992). Verbal hallucinations in normals, I: People who hear 'voices'. Applied Cognitive Psychology, 6, 379-387.

Bentall, R. P. (1990). The illusion of reality: a review and integration of psychological research on hallucinations. Psychological Bulletin, 107, 82-95. 
Bentall, R. P. (2000). Hallucinatory experiences. En: E. Cardeña, S. J. Lynn \& S. Krippner (Eds.). Varieties of anomalous experience: Examining the scientific evidence (pp. 85-120). Washington, DC: American Psychological Association.

Berenbaum, H.; Kerns, J. \& Raghavan, C. (2000). Anomalous experiences, peculiarity, and psychopathology. En: E. Cardeña, S. J. Lynn \& S. Krippner (Eds.). Varieties of anomalous experience: Examining the scientific evidence (pp. 25-46). Washington, DC: American Psychological Association.

Bernstein, E. M. \& Putnam, F. W. (1986). Development, reliability, and validity of a dissociation scale. Journal of Nervous and Mental Disease, 174, 727-735.

Bourguignon, E. (1976). Possession. San Francisco: Chandler y Sharp Publishers.

Bremner, S. D.; Southwick, S.; Brett, E.; Fontana, A.; Rosenheck, R. \& Charney, D. S. (1992). Dissociation and posttraumatic stress disorder in Vietnam combat veterans. American Journal of Psychiatry, 149, 328-332.

Burges Watson, I. P.; Hoffman, L. \& Wilson, G. V. (1988). The neuropsychiatry of post-traumatic stress disorder. British Journal of Psychiatry, 152, 164-173.
Butler, L. D.; Duran, R. E. F.; Jasiukaitis, P.; Koopman, C. \& Spiegel, D. (1997). Hypnotizability and traumatic experience: a diathesis-stress model of dissociative symptomatology. American Journal of Psychiatry, 153, 42-63.

Cardeña, E. (1994). The domain of dissociation. En: S. J. Lynn \& J. W. Rhue (Eds.). Dissociation: Clinical and theoretical perspectives ( $\mathrm{pp}$. 15-31). Nueva York: Guilford.

Carlson, E. B. (1994). Studying the interaction between physical and psychological states with the Dissociative Experiences Scale. En: D. Spiegel. (Ed.). Dissociation: Culture, mind, and body. Washington, DC: American Psychiatric Press.

Castillo, R. J. (1997). Dissociation. En: W. S. Tseng \& J. Streltzer (Eds.). Culture psychopathology (pp. 101123). Nueva York: Brunner/Mazel.

Couch, A. \& Keniston, K. (1960). Yeasayers and naysayers: Agreeing response set as a personality variable. Journal of Abnormal and Social Psychology, 60, 151-174.

Council, J. R. \& Green, J. P. (2004). Examining the Absorption-Hypnotizability Link: The Roles of Acquiescence and Consistency Motivation. International Journal of Clinical and Experimental Hypnosis, 52, 364-377. 
Crawford, H. J.; Brown, A. M. \& Moon, C. E. (1993). Sustained attentional and disattentional abilities: differences between low and highly hypnotizable persons. Journal of Abnormal Psychology, 102, 534-543.

Destun, L. M., \& Kuiper, N. A. (1999). Phenomenal characteristics associated with real and imagined events: the effects of event valence and absorption. Applied Cognitive Psychology, 13, 175-186.

Eisen, M. L., \& Carlson, E. B. (1998). Individual differences in suggestibility: Examining the influence of dissociation, absorption, and a history of childhood abuse. Applied Cognitive Psychology, 12, S47-S61.

Freyd, J. J.; Martorello, S. R.; Alvarado, J. S.; Hayes, A. E. \& Christman, J. C. (1998). Cognitive environments and dissociative tendencies: Performance on the standard Stroop task for high versus low dissociators. Applied Cognitive Psychology, 12, S91-S103.

Frischholz, E. J.; Braun, B. G.; Sachs, R. G.; Schwartz, D. R.; Lewis, J.; Shaeffer, D.; Westergaard, C. \& Pasquotto, J. (1992). Construct validity of the dissociative experiences scale: II. Its relationship to hypnotizability. American Journal of Clinical Hypnosis, 35, 145-152.
Galton, F. (1883). Inquiries into human faculty and its development. Londres: Macmillan.

Garry, M.; Manning, C. G.; Loftus, E. F. \& Sherman, S. J. (1996). Imagination inflation: imagining a childhood event inflates confidence that it occurred. Psychonomic Bulletin y Review, 3, 208-214.

Glicksohn, J. (1990). Belief in the paranormal and subjective paranormal experience. Personality and Individual Differences, 11, 675-683.

Glicksohn, J. (1993-4). Rating the incidence of an altered state of consciousness as a function of the rater's own absorption score. Imagination, Cognition and Personality, 13, 225228.

Glicksohn, J. (2001). Temporal cognition and the phenomenology of time: A multiplicative function for apparent duration. Consciousness and Cognition, 10, 1-25.

Glicksohn, J., \& Avnon, M. (19971998). Explorations in virtual reality: absorption, cognition and altered state of consciousness. Imagination, Cognition and Personality, 17, 141151.

Glicksohn, J.; Salinger, O. \& Roychman, A. (1992). An exploratory study of syncretic experience: eidetics, synaesthesia and absorption. Perception, 21, 637-642. 
Glickshon, J.; Steinbach, I. \& Elimalach-Malmilyan, S. (1999). Cognitive dedifferentiation in eidetics and synaesthesia: Hunting for the ghost once more. Perception, 28, 109120.

Glickson, J. \& Barret, T. R. (2003). Absorption and hallucinations. Applied Cognitive Psychology, 17, 833-849.

Glisky, M. L.; Tataryn, D. J.; Tobias, B. A.; Kihlstrom, J. F. \& McConkey, K. M. (1991). Absorption, openness to experience, and hypnotizability. Journal of Personality and Social Psychology, 60, 263-272.

Golub, D. (1995). Cultural variations in multiple personality disorder. En: L. Cohen, J. Berzoff \& M. Ellin (Eds.). Dissociative identity disorder (pp. 285-327). Nortirvale, NJ.: Aronson.

Goodman, E. (1988). How about demons? Indiana: Indiana University Press.

Grady, K. E. \& Wallston, B. S. (1988). Research in health care setting. Newbury Park: Sage.

Hilgard, E. R. (1986). Divided consciousness. New York: Wiley.

Hoffman, R. E. (1986). Verbal hallucinations and language production processes in schizophrenia. Behavioral and Brain Sciences, 9, 503548.
Horowitz, M. J. (1986). Stress response syndromes (2da. edición). Northvale, NJ: Jason Aronson.

Horselenberg, R.; Merckelbach, H.; Muris, P.; Sijsenaar, M. \& Spaan, V. (2000). Imagining fictitious childhood events: the role of individual differences in imagination inflation. Clinical Psychology and Psychotherapy, 7, 128-137.

Hyman, I. E. \& Billings, F. J. (1998). Individual differences and the creation of false memories. Memory, 6, 120.

James, W. (1892). Psychology: Briefer Course. Nueva York: Henry Holt.

Kihlstrom, J. E, \& Hoyt, L. P. (1990). Repression, dissociation, and hypnosis. En: J. L. Singer (Ed.). Repression and dissociation (pp. 181208). Chicago: University of Chicago Press.

Kihlstrom, J. (1994). Comentary: Psychodynamics and social cognition. Notes on the fusion of psychoanalysis and psychology. Journal of Personality, 62, (4), 681.

Kim, J. \& Mueller, C. W. (1978). Factor analysis: Statistical methods and practical issues. Newbury Park: Sage.

Kinzie, J. D. (1989). Post-traumatic stress disorder. En: H. I. Kaplan \& B. J. Sadock (Eds.). Comprehensive textbook of psychiatry, Vol. 1, 5. ${ }^{\mathrm{a}}$ 
edición (pp. 1000-1008). Baltimore: Williams y Wilkins.

Launay, G., y Slade, P. (1981). The measurement of hallucinatory predisposition in male and female prisoners. Personality and Individual Differences, 2, 221-234.

Lawrence, J. R. \& Perry, C. (1983). Hypnotically created memory among highly hypnotizable subjects. Science, 222, 523-524.

Lawrence, T.; Edwards, C.; Barraclough, N.; Church, S., \& Hetherington, F. (1995). Modelling childhood causes of paranormal belief and experience: childhood trauma and childhood fantasy. Personality and Individual Differences, 19, 209215.

Loftus, E. F. (1997). Creating childhood memories. Applied Cognitive Psychology, 11, 75-86.

Lynn, S. J., \& Rhue, J. W. (1988). Fantasy proneness: Hypnosis, developmental antecedents, and psychopathology. American Psychologist, 43, 35-44.

Martínez-Taboas, A. (1999). A case of spirit possession and glossolalia. Culture, Medicine and Psychiatry, 23, 333-348.

Martínez-Taboas, A. (2001). Dissociative experiences and disorders: A review. International Journal of Parapsychology, 12, 131-162.
Merckelbach, H.; Muris, P.; Horselenberg, R. \& Stougie, S. (2000). Dissociative experiences, response bias, and fantasy proneness in college students. Personality and Individual Differences, 28, 49-58.

Messick, S. (1994). The matter of style: manifestations of personality in cognition, learning, and teaching. Educational Psychologist, 29, 121136.

Nachson, I. (2001). Truthfulness, deception and self-deception in recovering true and false memories of child sexual abuse. International Review of Victimology, 8, 1-18.

Pitblado, C., \& Sanders, B. (1991). Reliability and short term stability of scores on the Dissociative Experiences Scale. In B. Braun (Ed.), Proceedings of the Eighth International Conference on Multiple Personality/Dissociative States ( $\mathrm{p}$. 19). Chicago, IL: Rush Presbyterian-St. Luke's Medical Center.

Platt, R. D.; Lacey, S. C.; Iobst, A. D. \& Finkelman, D. (1998). Absorption, dissociation, and fantasy-proneness as predictors of memory distortion in autobiographical and laboratory-generated memories. Applied Cognitive Psychology, 12, S77-S89.

Posey, T. B., \& Losch, M. E. (1983). Auditory hallucinations of hearing voices in 375 normal subjects. 
Imagination, Cognition and Personality, 3, 99-113.

Putnam, E. W. (1997). Dissociation in children and adolescents. Nueva York: Guilford.

Putnam E. W. \& Carlson, E. B. (1998). Hypnosis, dissociation, and trauma: Myths, metaphors, and mechanisms. En: J. D. Bremner \& C. R. Marmar (Eds.). Trauma, memory and dissociation (pp. 27-56), Washington, DC: American Psychiatric Press.

Rader, C., \& Tellegen, A. (1987). An investigation of synesthesia. Journal of Personality and Social Psychology, 52, 981-987.

Ray, W. J. (1996). Dissociation in normal populations. En: L.Y. Michelson \& W. J. Ray (Eds.). Handbook of dissociation (pp. 51-66). Nueva York: Plenum.

Roche, S. M., \& McConkey, K. M. (1990). Absorption: nature, assessment, and correlates. Journal of Personality and Social Psychology, 59, 91-101.

Ross, C. A. (1989). Multiple personality disorder. Diagnosis, clinical feature, and treatment. Nueva York: Wiley.

Ross, C. A.; Norton, G. R. \& Anderson, G. (1988). The Dissociative Experiences Scale: A replication study. Dissociation, 1(3), 21-22.
Ross, C. A.; Joshi, S. \& Currie, R. (1991). Dissociative experiences in the general population: A factor analysis. Hospital and Community Psychiatry, 42, 297-301.

Ross, C. A.; Ellason, J. W. \& Anderson, G. (1995). A factor analysis of the Dissociative Experiences Scale (DES) in dissociative identity disorder. Dissociation, 8, 229-235.

Sarbin, T. R., \& Juhasz, J. B. (1967). The historical background of the concept of hallucination. Journal of the History of the Behavioral Sciences, 5, 339-358.

Sargant, W. (1957). Battle for the mind: A physiology of conversion and brain-washing. Nueva York: Doubleday.

Sargant, W. (1973). The mind possessed: A physiology of possession, mysticism and faith healing. Londres: Heinemann.

Sidgwick, H. (1894). Report of the census of hallucinations. Proceedings of the Society for Psychical Research, 10, 25-422.

Slade, P. D. \& Bentall, R. P. (1988). Sensory deception: A scientific analysis of hallucination. Baltimore, MD: The Johns Hopkins University Press.

Spiegel, D.; Hunt, T. \& Dondershine, H. E. (1988). Dissociation and hypnotizability in posttraumatic stress 
disorder. American Journal of Psychiatry, 145, 301-305.

Steinberg, M. (1995). Handbook for the assessment of dissociation. Washington, DC: American Psychiatric Press.

Tellegen, A. (1982). Multidimensional Personality Questionnaire. University of Minnesota.

Tellegen, A. \& Atkinson, G. (1974). Openness to absorbing and selfaltering experiences ('absorption'), a trait related to hypnotic susceptibility. Journal of Abnormal Psychology, 83, 268-277.

Van der Kolk, B. \& Van der Hart, O. (1989). Pierre Janet and the breakdown of adaptation in psychologi- cal trauma. American Journal of Psychiatry, 146, 1530-1540.

Waller, N. G.; Putnam, E. W. \& Carlson, E. B. (1996). Types of dissociation and dissociative types: A taxometric analysis of dissociative experiences. Psychological Methods, 1, 300-321.

West, D. J. (1948). A mass-observation questionnaire on hallucinations. Journal of the Society for Psychical Research, 34, 187-196.

Wilson, S. C. \& Barber, T. X. (1983). The fantasy-prone personality: implications for understanding imagery, hypnosis, and parapsychological phenomena. En: A. A. Sheikh (Ed.). Imagery: Current theory, research, and application (pp. 340-382). Nueva York: John Wiley. 


\section{ANEXO \\ Cuestionario de experiencias alucinatorias Nunca $=0 ;$ Rara vez $=1 ;$ Ocasionalmente $=2 ;$ A menudo $=3 ;$ Muy a menudo $=4$}

1. He oído que alguien decía mi nombre. Por ejemplo, al pasar al lado de gente desconocida, aunque sabía que realmente no me habían llamado y seguí adelante como si nada.

2. Cuando estoy completamente solo en casa, oigo una voz que me llama por mi nombre, una sola vez. Por ejemplo: "Carmen".

3. He oído una voz o varias voces que dicen lo que estoy haciendo y me lo repiten una y otra vez. Estas voces a veces tienen un tono agresivo y recriminatorio.

4. El verano pasado estaba en el jardín y de repente oí que mi mamá me llamaba desde dentro de casa. Su voz sonaba como si algo malo hubiera ocurrido, su voz era alta y clara. Corrí al interior pero no había nadie. Ella estaba afuera, en la calle, y no me había llamado.

5. He oído mis propios pensamientos en voz alta. En realidad los oigo como desde fuera de mi cabeza, a pesar de que estoy seguro de no haber hablado en voz alta.

6. He tenido la experiencia de oír la voz de Dios; pero no como si hablara en mi corazón sino como una voz que realmente viene desde fuera de mi cabeza.

7. He oído mi propia voz procedente de detrás de mí, en forma de frases cortas, normalmente tranquilizadoras, como "todo va bien" o "cálmate".

8. Puedo oír la voz de alguien conocido que me habla, sin estar presente. No es que me esté imaginando su voz, sino que realmente puedo oírla. Su voz me parece tan real que cuando esto sucede en ocasiones llego a contestarle.

9. Cuando estoy solo oigo voces de niños angustiados.

10. Por la noche oigo pasos, oigo respirar, tropezar, raspar, girar los picaportes de las puertas, puertas que se abren o se cierran, ventanas que son forzadas; pero cuando me levanto para mirar, no encuentro a nadie.

11. He tenido la experiencia de oír botellas romperse, o platos caer, o explosiones de aparatos domésticos (u otras cosas en mi casa), pero cuando voy en dirección a aquellos ruidos, todo está en su lugar.

12. Solo cuando me estoy durmiendo o estoy despertando del sueño, he oído voces o diálogos, música o melodías, sonidos, a veces agradables y otras no, pero que escucho nítidamente. 
13. Algunas de mis creaciones (dibujos, canciones, poesías, narrativas, etcétera) o mis principales inspiraciones creativas son en realidad producto de alguien externo, no presente, que me las dicta.

14. Me ha ocurrido oír nítidamente el diálogo de dos o más voces de personas como si estuvieran a mi lado. Estaban hablando en voz alta, pero no estaban físicamente donde yo me encontraba.

15. Cuando estoy solo, mantengo una agradable conversación en voz alta con un pariente o amigo fallecido y prácticamente siempre oigo lo que me dice.

16. Puedo, si lo deseo, mantener conversaciones con ángeles, el demonio o cualquier otra entidad espiritual, y escuchar claramente su voz como si estuvieran hablándome al oído.

17. Mientras viajaba por la ruta, estando en compañía de otras personas, he visto claramente a una persona parada, vestida y de buen aspecto. Pero yo fui el único que lo vio.

18. Cuando conduzco por la noche o camino por una calle, he visto algo a un lado de la carretera, como un perro; pero cuando me vuelvo para mirar no hay nada.

19. He visto un rostro rodeado de un brillo singular, que cuando aparece me da consejos, es muy amable y gentil.

20. Por la noche he visto cosas que se mueven a mi alrededor y dan toda la impresión de que hay alguien en mi habitación, aunque sé que no hay nadie.

21. He tenido la experiencia de ver claramente una figura con forma humana ante mí; alguien que no estaba físicamente presente en aquel momento. Incluso tuve la sensación de que tenía alguna intención hacia mí.

22. He tenido la experiencia de ver seres "elementales", como personas diminutas que reinan en la naturaleza. No tengo la sensación de que esas presencias estén allí, yo las veo claramente y veo cómo actúan.

23. Espontáneamente he visto a una persona a pesar de que sé que esa persona estaba fallecida. Además, estoy seguro de que esa presencia intentaba comunicarme algo.

24. He visto una luz o luces o campos de energía alrededor del cuerpo o de una parte del cuerpo de otra persona, lo cual -hasta donde pude determinar- no era debido a causas normales o naturales que yo pudiera explicar.

25. Solo cuando me estoy durmiendo o estoy despertando del sueño he visto sombras, figuras humanas o no humanas cerca de mi cama, yo las he visto claramente y veo lo que hacen. 
26. He tenido la sensación de estar bebiendo un refresco. Estaba sediento. Podía realmente saborearlo, aunque no lo estaba tomando.

27. Puedo saborear una comida. Si tengo hambre y pienso en esa comida, puedo saborearla aunque no la esté comiendo.

28. Solo cuando me estoy durmiendo o estoy despertando del sueño he tenido la experiencia de experimentar sabores en mi boca, agradables o desagradables, a pesar de que no he comido nada relacionado con esos sabores.

29. He tenido la experiencia de sentir una palmada en mi hombro, o cualquier otra sensación vívida de contacto físico de otra persona detrás de mí, pero cuando me doy vuelta no veo a nadie.

30. Antes de quedarme dormido, he sentido un aire frío que soplaba en mi cara. Era muy claro y de ninguna manera estaba dormido. Nadie estaba en la habitación salvo la persona que comparte mi cuarto, que estaba dormida. No sé de dónde pudo venir aquel aire frío.

31. He tenido la experiencia de despertarme porque alguien me sacudía el pie. Lo sentí como si ocurriera realmente. Me levanté para ver si había alguien, pero estaba yo solo en casa.

32. He tenido la experiencia de sentirme como 'fuera de mi cuerpo físico,' esto es, la sensación de que mi yo (mente, o conciencia o espíritu) estaba desplazada en un sitio diferente al de mi cuerpo.

33. Solo cuando me estoy durmiendo o estoy despertando del sueño he tenido las vívidas sensaciones de presencia, como si alguien o algo me tocara o presionara todo o alguna parte de mi cuerpo. Estas sensaciones me atemorizan.

34. He tenido la experiencia de oler algo que se quema, pero cuando trato de mirar qué puede ser, no encuentro nada en el fuego.

35. Olores nauseabundos y desagradables me invaden espontáneamente y a pesar de su intensidad, no puedo determinar de donde surgen.

36. Oler comidas fritas aunque no haya nada a mi alrededor es una experiencia que he tenido. Incluso se me hace 'agua la boca'.

37. He tenido la experiencia de oler vívidamente el perfume de una persona conocida por mí, a pesar de que no había ninguna explicación razonable por la cual ese aroma estaba presente donde yo me encontraba.

38. Solo cuando me estoy durmiendo o estoy despertando del sueño he tenido la experiencia de oler comidas o perfumes, o por el contrario, otros olores nauseabundos y desagradables, pero cuando trato de determinar el origen de esos olores no encuentro nada. 